Conference Proceedings Paper

\title{
Structure and Properties of Coatings Made of High-Entropy Alloy with Martensitic Transformations Obtained by HVOF Method in a Protective Atmosphere
}

\author{
Petr Rusinov *, Zhesfina Blednova \\ Kuban State Technological University, Moskovskaya Street 2, 350072 Krasnodar, Russia; blednova@mail.ru \\ * Correspondence: ruspiter5@mail.ru; Tel.: +7-908-690-8810
}

Received: 30 March 2020; Accepted: 25 April 2020; Published: 26 April 2020

\begin{abstract}
The article is devoted to the comprehensive studies of high-entropy CoCuTiZrHf coating obtained by HVOF in a protective atmosphere. We have examined metallophysical properties of the coating (using electron microscopy and X-ray diffraction analysis) in order to obtain new information about its structure, mechanical properties and phase composition. Functional properties were also determined. We studied the effect of mechanical activation of highly entropic material powder on the structural phase state and coating quality. The optimal technological parameters of $\mathrm{HVOF}$ in a protective atmosphere were determined for the formation of a highly entropic CoCuTiZrHf coating, which provides the highest adhesion and low porosity. We have developed statistical process models with parameter optimization. Based on complex metallophysical studies, the structure formation in highly entropic coatings was researched after HVOF in a protective atmosphere and subsequent heat treatment. Calorimetric tests of the CoCuTiZrHf alloy were performed to identify the exo-effect corresponding to the manifestation of phase transformation. We determined the mechanical properties of coated steel are.
\end{abstract}

Keywords: highly entropic materials; twinning; shape memory effect; mechanical activation; wear resistance.

\section{Introduction}

High-entropy alloys (HEA) have been the subject of intensive research in the last decade. Most of the HEA studies are focused on the structure of alloys, microstructural and mechanical properties [1]. However, few works are devoted to TRIP-effect [2-4] in these alloys. Alloys with shape memory effect (SME) have high strength, unique effects of single and multiple reversible thermomechanical memory, high level of reactive stresses, recovery and damping, high corrosion resistance and cyclic durability. All this makes them indispensable, despite the high cost, and determines the effective practical application as functional materials of the new generation. However, despite all the advantages, the high cost of the alloy limits their use in industry. Under these conditions, the direction related to methods of surface modification of steels by HEAs with SME becomes relevant. The formation of coatings using HEA with SME is currently carried out by various methods of high-energy impacts (argon-arc surfacing, laser surfacing with local protection, self-propagating high-temperature synthesis, various vacuum-plasma methods). We have developed a promising technology for producing HEAs with SME on the surface of products by high-velocity oxygen-fuel spraying (HVOF) in a protective atmosphere (argon medium) $[5,6]$.

The aim of the work is to study the structure and properties of the highly entropic CoCuTiZrHf coating with martensitic transformations with the development of optimal technological parameters 
for producing a HEA with the assessment of the specified structural, mechanical properties, which ensure the manifestation of phase transformation.

To accomplish the goal of the work, we have formulated the following research objectives. We have to study the laws of the mechanical activation effect of highly entropic material powder on the structural phase state and quality of the coating obtained by HVOF in a protective atmosphere. This ensures the functional and operational properties of the products. We have to develop the optimal technological parameters of HVOF in the protective atmosphere to form a highly entropic CoCuTiZrHf coating that provides reliable adhesion. On the basis of complex metallophysical studies it is also necessary to establish the patterns of structure formation in highly entropic coatings after HVOF in a protective atmosphere and subsequent heat treatment. We have to develop statistical models of the technological process with optimization of parameters and to reveal the effect of phase transformation during calorimetric tests of the alloy coating. We finally have to perform testing of steel samples with coatings from HEAs on "friction-wear" with justification of the coating material influence on improving the mechanical properties of products.

\section{Materials and Methods}

HVOF was sprayed on steel 1045. HVOF of mechanically activated Co17.6Cu18.8Ti9.6Zr18.3Hf35.7 wt.\%, powder was performed on a universal GLC-720 unit in argon medium on cylindrical samples (Ø10, sample length $50 \mathrm{~mm}$ ). Mechanical activation was carried out in an AGO-2U ball mill.

The microstructure was studied using a JSM-7500F ultra-high resolution scanning electron microscope and a JEM-2100 transmission electron microscope. Chemical analysis of the materials was carried out by an optical emission analyzer of metals and alloys Arcmet 8000 and an INCA $\mathrm{x}$-sight energy dispersive attachment model 7585 electron microscope JSM-7500F. The coating thickness was measured with a DMS 2E thickness gauge. X-ray phase analysis was performed on a Dron-7M device in $\mathrm{Cu}-\mathrm{K} \alpha$ radiation. The microhardness was measured on Falcon 503. The porosity of the coatings was determined by hydrostatic weighing [7]. Tests for wear of coated materials were performed on the installation "2070 SMT-1." Thermal analysis of materials was carried out on a NETZSCH STA 409 PC unit at heating and cooling rates of $10^{\circ} \mathrm{C} / \mathrm{min}$ to obtain a heating-cooling curve. Research methods: experimental and theoretical modeling, electron microscopy, X-ray diffraction analysis, multifractal parameterization of the structure, thermal calorimetry, mechanical tests.

\section{Results}

Before HVOF, mechanical activation of the powder was carried out. This helps to improve the quality of the coating and increase the strength properties, while there is a decrease in porosity and an increase in the adhesion of the coating to the base [6]. The particle size of the powder before mechanical activation is $25-50 \mu \mathrm{m}$ (Figure 1a,b). After mechanical activation it is 1-20 $\mu \mathrm{m}$ (Figure 1c,d). Powder particles after mechanical activation are in the form of deformed disks.

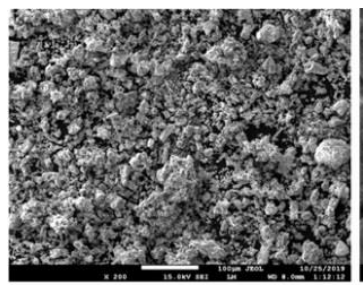

(a)

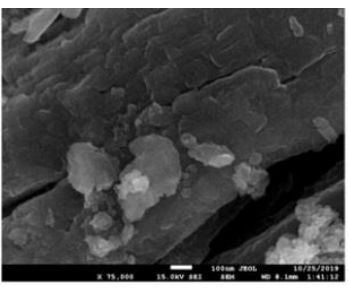

(b)

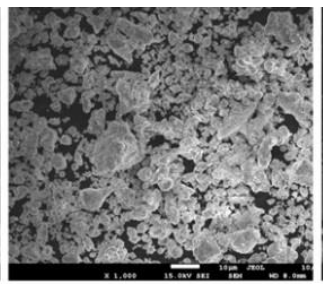

(c)

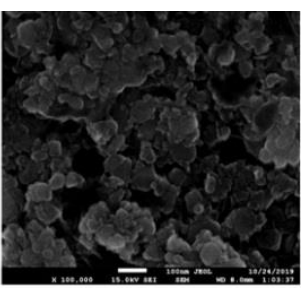

(d)

Figure 1. Morphology of Co17.6Cu18.8Ti9.6Zr18.3Hf35.7 powder particles: before mechanical activation, $\times 600-(\mathbf{a}) ; \times 75,000-(\mathbf{b})$; after mechanical activation (activation time $10 \mathrm{~min}) \times 1000-(\mathbf{c})$; $\times 100,000-(\mathbf{d})$. 
The technological process of forming a coating from a HEA with SME formed under phased high-energy influences includes the following stages: preparation and cleaning of the surface of the steel base, mechanical activation of metal powders, sequential HVOF coatings in a single technological cycle (if necessary, the powder can be deposited into different zones of the gas stream), subsequent thermal and thermomechanical treatment, optimization of process parameters, analysis and experimental verification of results with an assessment of the functional reliability of the composition "base-coating with SME". When testing the HVOF process, algorithmic planning and statistical processing were used at all stages. When searching for optimal HVOF modes using the experimental design method, a sequence of experiments was adopted that allows to use the gradient search methods with an unknown function, i.e., approximately restore the law of functioning of the object from experimental data $[5,8]$. When developing statistical models of the technological process, we used a uniform rotatable plan [9].

We estimated the temperature effect on the microstructure and properties of the surface layers in the contact zone between powder particles and the substrate during a high-impact effect. As a result of statistical processing we obtained empirical dependence equations of temperature $\left(T_{p}\right)$ and speed $\left(\mathrm{V}_{\mathrm{p}}\right)$ of mechanically activated powder particles (3-40 microns in size) on the spraying distance:

$$
\begin{gathered}
T_{p}=A-B \cdot L-C \cdot L^{2}, \\
V_{p}=A_{1}+B_{1} \cdot L-C_{1} \cdot L^{2},
\end{gathered}
$$

where L-the spraying distance; $\mathrm{A}, \mathrm{B}, \mathrm{C}, \mathrm{A}_{1}, \mathrm{~B}_{1}, \mathrm{C}_{1}$-coefficients obtained as a result of statistical processing of experimental data. The values of the coefficients $A, B, C, A_{1}, B_{1}, C_{1}$ are summarized in Table 1.

Table 1. The values of the coefficients.

\begin{tabular}{ccccccc}
\hline Particle Size, microns & A & B & C & A $_{1}$ & B $_{1}$ & $\mathbf{C}_{1}$ \\
\hline 3 & 2074.9 & 3.6 & 0.0005 & 879.3 & 0.41 & 0.0034 \\
10 & 2047.75 & 3.42 & 0.0005 & 580.76 & 1.64 & 0.0042 \\
20 & 2012.29 & 3 & 0.0009 & 346.3 & 1.12 & 0.0026 \\
30 & 1895.6 & 1.23 & 0.0031 & 218.5 & 1.46 & 0.0027 \\
40 & 1836.7 & 0.54 & 0.0035 & 146.77 & 1.2 & 0.002 \\
\hline
\end{tabular}

As we can see from Figure 2a, finer particles of mechanically activated powder are heated very quickly to the melting temperature and are cooled faster during the bombardment of the hardened surface. Larger powder particles (size 30-40 $\mu \mathrm{m}$ ) heat up much longer than small ones $(3-20 \mu \mathrm{m})$, but their temperature remains at a fairly high level for a long time.

The analysis of Figure $2 b$ shows that smaller particles of mechanically activated powder (up to $10 \mu \mathrm{m}$ in size), falling into a high-velocity oxygen-fuel jet, accelerate faster up to a distance of $\approx 120$ $\mathrm{mm}$ and then there is a sharp decrease in the speed of moving powder particles. The speed of larger powder particles varies insignificantly and remains at a fairly high level with an increase in the spraying distance. 


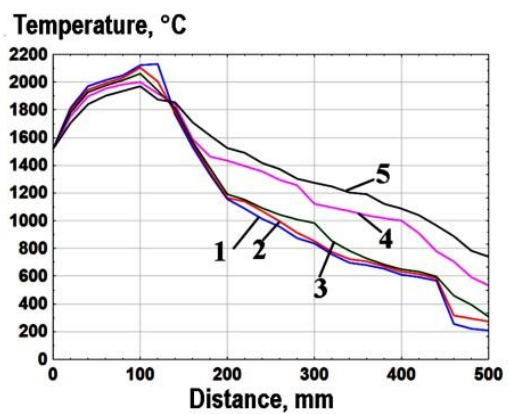

(a)

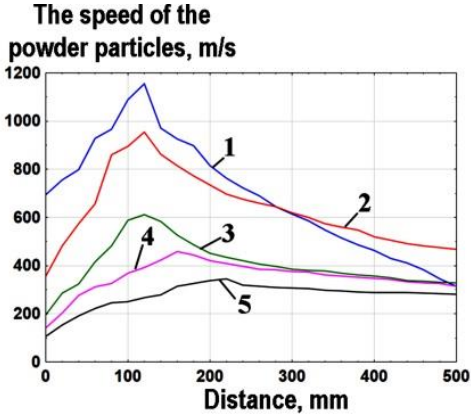

(b)

Figure 2. The dependence of temperature (a) and speed (b) of particles of mechanically activated powder on the spraying distance: particle size $3 \mu \mathrm{m}-1 ; 10 \mu \mathrm{m}-2 ; 20 \mu \mathrm{m}-3 ; 30 \mu \mathrm{m}-4 ; 40 \mu \mathrm{m}-5$.

Figure 3 shows the results of experimental-theoretical modeling of the temperature and speed of particles, mechanically activated powders in contact with the base, depending on the spraying distance.

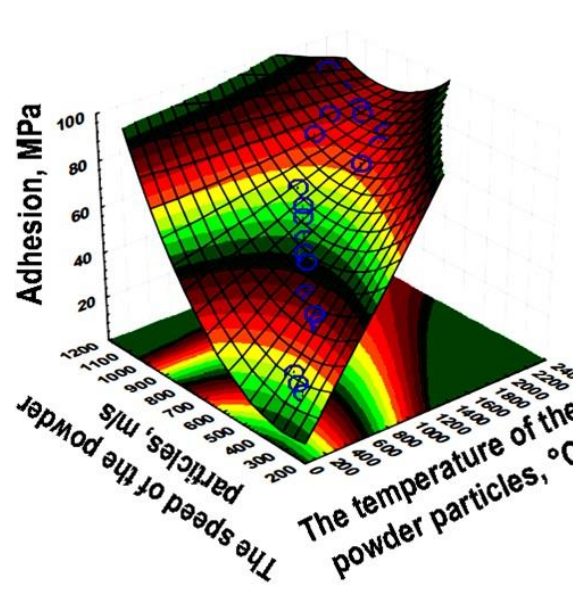

(a)

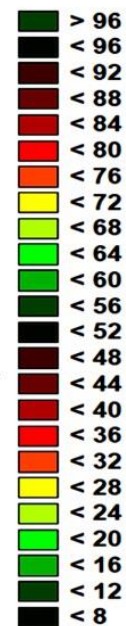

$<8$

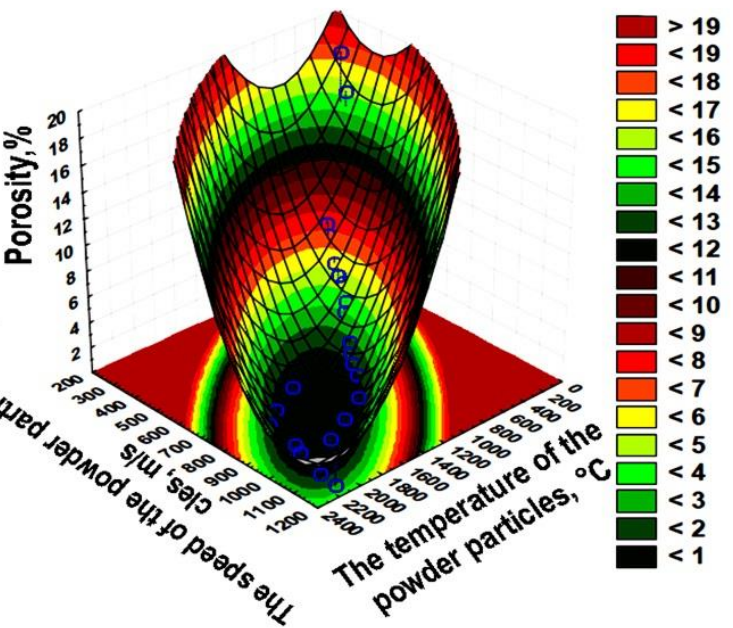

(b)

Figure 3. The dependence of temperature and speed of mechanically activated powder particles on HVOF distance: the adhesion strength of the coating to the base $-(\mathbf{a})$; porosity $-(\mathbf{b})$.

Metallographic studies of the CoCuTiZrHf coating obtained by HVOF of a mechanically activated powder showed that when the powder particles pass through a gas flame, they heat up and, upon impact, harden in the form of deformed disks. These disks have a diameter of $0.5-15 \mu \mathrm{m}$ and a thickness of 0.3-1.2 microns. After HVOF in a protective atmosphere, we performed annealing at a temperature of $1123 \mathrm{~K}$ for $1 \mathrm{~h}$. Annealing was carried out in order to eliminate the intermetallic phases CoTi, CuTi, HfTi formed in the coating after HVOF. Since these intermetallic phases worsen the phase transformation in the alloy.

The coating thickness was $1 \mathrm{~mm}$. After annealing, the austenitic structure of B2, CoCuTiZrHf alloy with a grain size of $25-125 \mathrm{~nm}$ is shown in Figure $4 \mathrm{a}, \mathrm{b}$. The martensitic structure of B19', the corresponding microelectron diffraction pattern, is shown in Figure $4 \mathrm{c}, \mathrm{d}$. In many ways, the formation of such a coating is associated with the features of HVOF. Grain grinding leads to a decrease in pore volume concentration by $10-15 \%$ and the formation of denser interfaces. Studies on the assessment of pore size and their percentage showed that the pore content in the coating is less than $1 \%$, the adhesion of the coating was $78 \mathrm{MPa}$. The adhesion of similar coatings obtained by HVOF in a protective atmosphere without mechanical activation was $52 \mathrm{MPa}$, the porosity was $7 \%$. Macroanalysis of the coating obtained by the proven technology showed that the coating structure is quite dense, with a minimum pore content and size. The interface between the coating and the substrate is without visible cracks. 


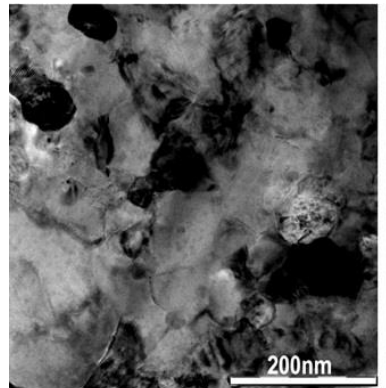

(a)

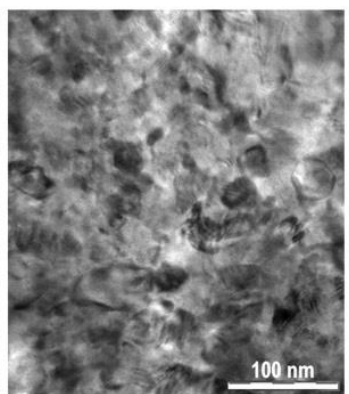

(b)

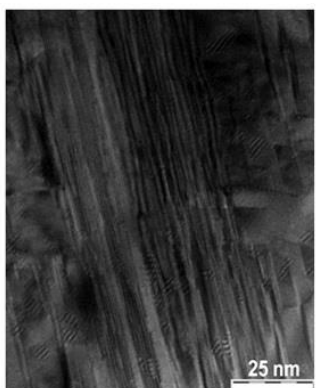

(c)

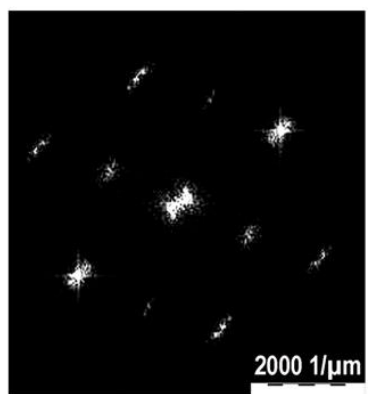

(d)

Figure 4. Microstructures of CoCuTiZrHf coatings obtained by HVOF in a protective atmosphere: austenitic structure, (a) $\times 100,000$; (b) $\times 120,000$; martensitic structure, (c) $\times 120,000$; microelectron diffraction pattern corresponding to the martensitic phase $-(\mathbf{d})$.

At room temperature, the main structural components of the CoCuTiZrHf coating obtained by HVOF in a protective atmosphere are as follows: the austenitic B2 phase with a cubic lattice, the martensitic B19' phases with a monoclinic lattice, the HfTi phase with a hexagonal lattice, a small CoTi phase, CuTi with a tetragonal lattice, as well as titanium oxide (TiO) of less than $2 \%$ (Figure 5a). After heat treatment (annealing at a temperature of $1123 \mathrm{~K}$ ) we observed: an austenitic B2 phase with a cubic lattice, martensitic B19' phases with a monoclinic lattice, an HfTi phase with a hexagonal lattice, and a small amount of titanium oxide (TiO) of less than $1 \%$ (Figure 5b).

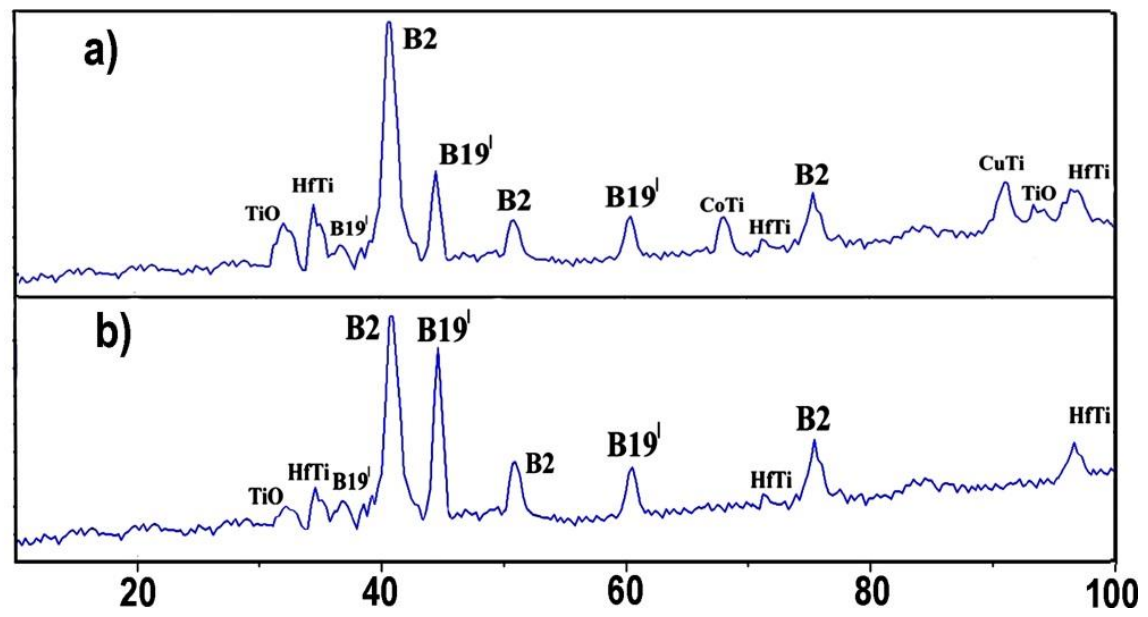

Figure 5. Diffraction patterns of CoCuTiZrHf: obtained by HVOF in a protective atmosphere (argon medium) - (a); after heat treatment (annealing at a temperature of $1123 \mathrm{~K})-(\mathbf{b})$.

As we can see from the X-ray phase analysis (Figure 5a), the CoCuTiZrHf coating after HVOF in the protective atmosphere is almost in the austenitic state. In the second main phase, CoCuTiZrHf in significant quantities is the martensitic phases of B19'. Intermetallic phases HfTi, CoTi, CuTi are also present in the layer (Table 2).

After annealing (argon medium) in the high-entropy CoCuTiZrHf coating, we observe the absence of CoTi, CuTi intermetallic phases and a decrease in the intensity of the HfTi and TiO intermetallic phases. Apparently, the CoTi, CuTi phases dissolve upon annealing in the CoCuTiZrHf alloy. 
Table 2. Parameters of the crystal lattices of the phases making up the Co-Cu-Ti-Zr-Hf coating.

\begin{tabular}{cccccc}
\hline Phase & $\mathbf{a}, \mathbf{n m}$ & $\mathbf{V}_{\mathbf{a t}} \cdot \mathbf{1 0}$ & $\mathbf{b}, \mathbf{n m}$ & $\mathbf{c}, \mathbf{n m}$ & $\boldsymbol{\beta}$, degrees \\
\hline B2 (cubic) & 0.3021 & 27.57 & - & - & 90.00 \\
B19' (monoclinic) & 0.4651 & 55.56 & 0.4112 & 0.2905 & 98.00 \\
CuTi (tetragonal) & 0.316 & 26.86 & 0.316 & 0.269 & 90.00 \\
CoTi (tetragonal) & 0.3971 & 59.8 & 0.3971 & 0.3792 & 90.00 \\
HfTi (hexagonal) & 0.305 & 45.21 & 0.305 & 0.486 & 90.00 \\
TiO (monoclinic) & 0.587 & 229.11 & 0.936 & 0.417 & 108.12 \\
\hline
\end{tabular}

To identify the phase transformation temperatures, calorimetric studies of the CoCuTiZrHf alloy after HVOF were carried out, followed by heat treatment (Figure 6).

As can be seen from the results of calorimetric studies (Figure 6), the phase transformation in the CoCuTiZrHf alloy is reversible $(\mathrm{M} \leftrightarrow \mathrm{A}), \mathrm{B} 19^{\prime} \leftrightarrow \mathrm{B} 2$.

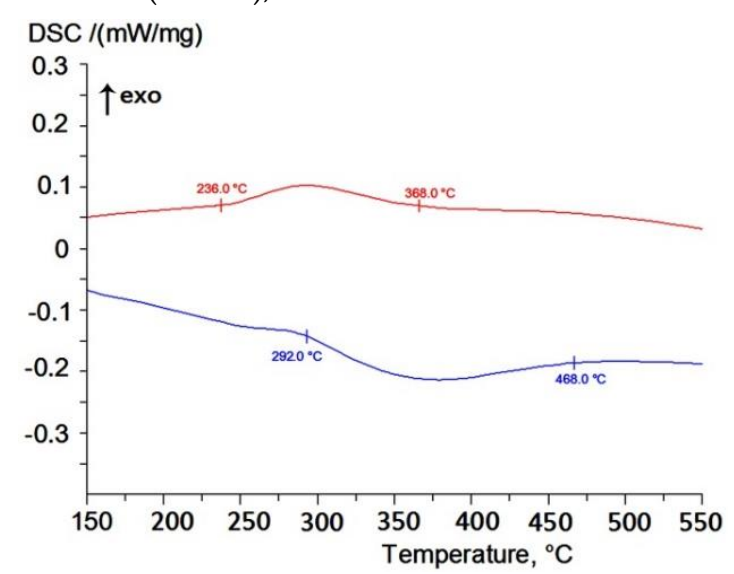

Figure 6. Calorimetric heating-cooling curves of the CoCuTiZrHf alloy after HVOF followed by heat treatment.

The distribution of microhardness over the thickness of the CoCuTiZrHf coating is uneven, in the range of $398-472 \mathrm{~kg} / \mathrm{mm}^{2}$ (after HVOF in a protective atmosphere) (Figure 7a, curve 1); 320-372 $\mathrm{kg} / \mathrm{mm}^{2}$ (after HVOF in a protective atmosphere followed by heat treatment) (Figure 7a, curve 2). The decrease in the microhardness of the CoCuTiZrHf coating after the heat treatment is explained by the dissolution of the intermetallic phases (Figure $5 b$ ) and a small increase in the grain structure of the coating (Figure $4 a, b$ ). The uneven distribution of microhardness over the coating thickness is explained by the predominant presence of two types of austenite-martensite structure. The microhardness of the steel base is in the range $175-203 \mathrm{~kg} / \mathrm{mm}^{2}$ (Figure 7).

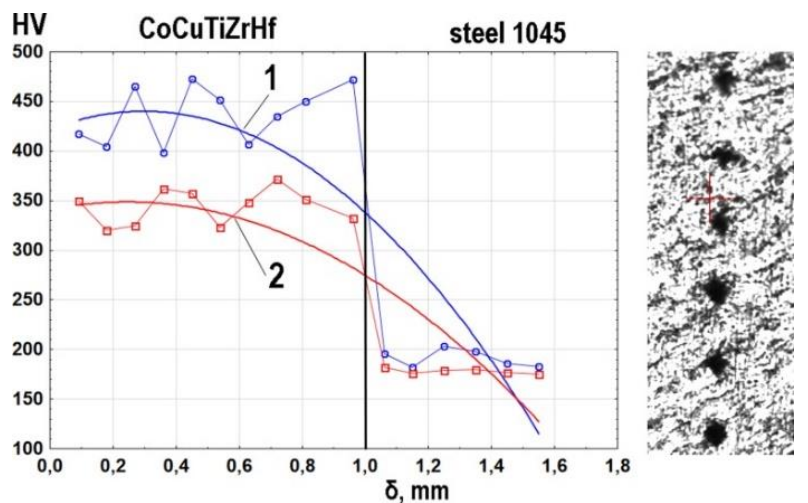

Figure 7. The distribution of microhardness “Co-Cu-Ti-Zr-Hf - steel 1045" by thickness: 1 - after HVOF in a protective atmosphere; 2 - after HVOF in a protective atmosphere, followed by heat treatment. 
The dependence of the microhardness HV0.1 of the coating on the thickness $(\delta)$ is described by Equations (3) and (4). Equation (3) corresponds to curve 1, Figure 7, and Equation (4) of curve 2, Figure 7.

$$
\begin{aligned}
& \text { HV } 0.1=421.79+123.21 \delta-206.97 \delta^{2},\left(\mathrm{~kg} / \mathrm{mm}^{2}\right) \\
& \text { HV } 0.1=340.86+64.06 \delta-130.18 \delta^{2},\left(\mathrm{~kg} / \mathrm{mm}^{2}\right)
\end{aligned}
$$

where $\delta$ - coating thickness, $\mathrm{mm}$.

Testing of steel with a high-entropy coating with SME for wear was carried out with dry friction of the sample coated on a hard rotating disk on a 2070 SMT-1 testing machine at a disk rotation speed of $\mathrm{v}=0.5 \div 2 \mathrm{~m} / \mathrm{s}$ and pressure $\mathrm{P}=2 \div 12 \mathrm{MPa}$, with registration of temperature in the contact zone (Figure 8a). The surface relief of samples with coatings from HEAs after testing is shown in Figure $8 \mathrm{~b}$. The wear rate was estimated based on the processing of experimental data using the Statistica v10.0 application package in the SPSS environment.

In Figure 8c, we presented the results of the wear test of steel 1045 samples with a highly entropic CoCuTiZrHf coating $1 \mathrm{~mm}$ thick, obtained by HVOF in a protective atmosphere according to the "disk-disk" scheme. The tests were carried out under dry friction at various slip speeds. During loading, we measured the temperature at the point of contact.

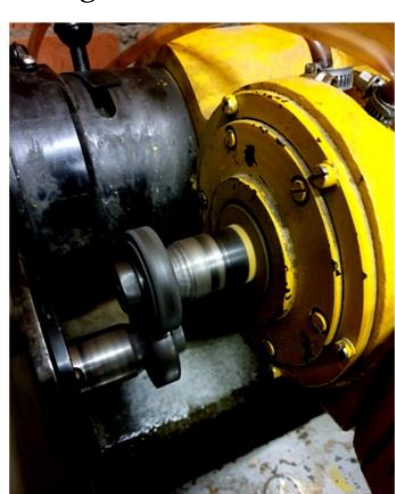

(a)

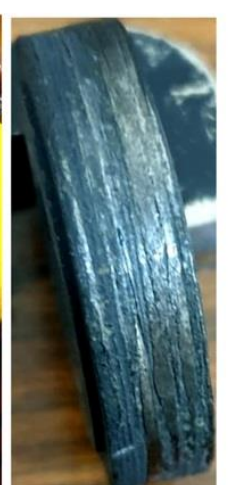

(b)

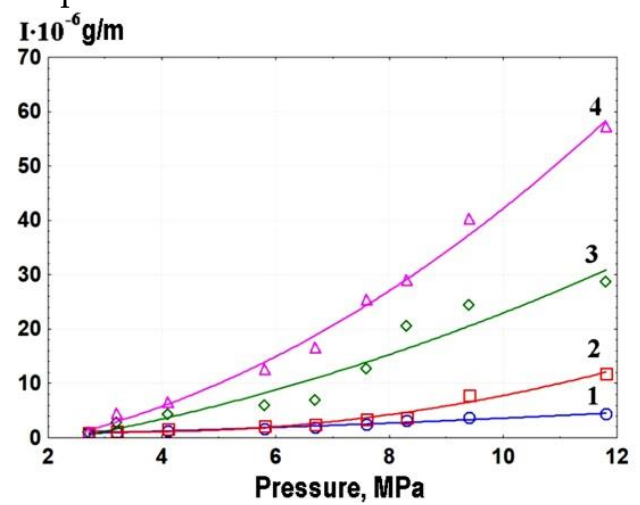

(c)

Figure 8. The process of testing the friction-wear of samples with coatings from HEAs on a testing machine 2070 SMT-1 - (a); surface relief of samples with coatings from HEAs after testing - (b); the dependence of the wear rate I on the disk pressure $\mathrm{P}$ with CoCuTiZrHf coating, the sliding speed of the disk is $0.5 \mathrm{~m} / \mathrm{s}-1 ; 1 \mathrm{~m} / \mathrm{s}-2 ; 1.5 \mathrm{~m} / \mathrm{s}-3 ; 2 \mathrm{~m} / \mathrm{s}-4-$ (c).

The empirical dependences of the wear rate (I) on the disk pressure (P) for the CoCuTiZrHf alloy are described by Equations (5)-(8):

$$
\begin{gathered}
\mathrm{I}=0.1364+0.2224 \cdot \mathrm{P}+0.0127 \cdot \mathrm{P}^{2}(\text { disk slip speed } 0.5 \mathrm{~m} / \mathrm{s}), \\
I=3.264-1.1816 \cdot \mathrm{P}+0.1634 \cdot \mathrm{P}^{2}(\text { disk slip speed } 1 \mathrm{~m} / \mathrm{s}), \\
\mathrm{I}=-3.8309+1.2502 \cdot \mathrm{P}+0.143 \cdot \mathrm{P}^{2}(\text { disk slip speed } 1.5 \mathrm{~m} / \mathrm{s}), \\
I=-3.596+0.8539 \cdot \mathrm{P}+0.3724 \cdot \mathrm{P}^{2}(\text { disk slip speed } 2 \mathrm{~m} / \mathrm{s}) .
\end{gathered}
$$

An analysis of the test results shows that, at high test speeds, steel 1045 with coatings have almost no running-in period, and at low speeds it is weakly expressed. The increase in wear resistance of steel 1045 with a high-entropy coating CoCuTiZrHf is 2.32 times compared with that of steel 1045.

\section{Discussion}

During the wear test of samples with composite coatings, we observed an increase in surface temperature in the friction zone. Sufficiently high pressures arising from friction cause transformation plasticity due to the formation of stress martensite. The combination of these 
processes determines the wear resistance of the sample. The increase in wear resistance after a full treatment cycle is explained by reversible plastic deformations (1.6\%). Experience shows [6] that the higher the reversible deformations, the higher the wear resistance. The formation of a nanostructured state upon surface modification gives the composite the properties of dynamic adaptation to operating conditions. Thus, the considered metal composite "base - layer made of a material with martensitic transformations" can be attributed to the number of smart, self-adaptive, widely used ones in aerospace engineering, which actively counteracts external influences, reduces vibration influence and redistributes mechanical stresses.

\section{Conclusions}

We studied the mechanical activation effect of a highly entropic material powder on the structural phase state and the quality of the coating obtained by HVOF in a protective atmosphere. It was shown that the use of mechanical activation reduces the porosity of the coating (the pores are less than $1 \%$ ), increases the adhesion of the coating up to $78 \mathrm{MPa}$. Without mechanical activation of the powder the adhesion amounted up to $52 \mathrm{MPa}$, porosity was $7 \%$. As a result of the experiments, the optimal technological parameters of HVOF in a protective atmosphere were determined in order to form the highly entropic CoCuTiZrHf coating. The coating provides the highest adhesion of 78 MPa and low porosity of less than $1 \%$. We have developed statistical models of technological process with optimization of parameters such as spraying distance, speed and temperature of powder particles. Based on complex metallophysical studies we have researched the structure formation in highly entropic coatings after HVOF in a protective atmosphere and subsequent heat treatment. Since the grain size in the coating was $25-125 \mathrm{~nm}$, the structure is mainly austenite-martensite. During calorimetric tests of the CoCuTiZrHf alloy, we observed a significant exo-effect corresponding to the manifestation of the B19‘ $\leftrightarrow \mathrm{B} 2$ phase transformation. We have established experimentally that after HVOF in a protective atmosphere of a mechanically activated SME powder based on CoCuTiZrHf, the wear resistance of the coating increases by 2.32 times compared to that of steel 1045.

\section{Patents}

As part of the development of a technology for spraying high-entropy powder materials, a "Installation for producing nanostructured composite multifunctional coatings from a material with a shape memory effect on the part surface" was developed (a positive decision was received on the grant of a patent for the invention No. 2019137463 of 02/14/2020).

Funding: The work was performed as part of a grant from the Russian Science Foundation (Agreement No. 19-19-00331).

Conflicts of Interest: Authors declares no conflict of interest.

\section{References}

1. Löbel, M.; Lindner, T.; Mehner, T.; Lampke, T. Microstructure and Wear Resistance of AlCoCrFeNiTi High-Entropy Alloy Coatings Produced by HVOF. Coatings 2017, 7, 144, doi:10.3390/coatings7090144.

2. Soares, R.L.; De Castro, W.B. Effects of composition on transformation temperatures and microstructure of Ni-Ti-Hf shape memory alloys. REM - Int. Eng. J. 2019, 72, 227-235, doi:10.1590/0370-44672018720072.

3. Exarchos, D.A.; Dalla, P.-A.; Tragazikis, I.K.; Dassios, K.; Zafeiropoulos, N.E.; Karabela, M.M.; De Crescenzo, C.; Karatza, D.; Musmarra, D.; Chianese, S.; et al. Development and Characterization of High Performance Shape Memory Alloy Coatings for Structural Aerospace Applications. Materials 2018, 11, 832, doi:10.3390/ma11050832.

4. Lee, H.-C.; Chen, Y.-J.; Chen, C.-H.; Lee; Chen Effect of Solution Treatment on the Shape Memory Functions of (TiZrHf)50Ni25Co10Cu15 High Entropy Shape Memory Alloy. Entropy 2019, 21, 1027, doi:10.3390/e21101027.

5. Blednova, Z.M.; Rusinov. P.O. Advances in Nanotechnology: Collective Scientific Monograph. Formation of Nanostructured Blankets from Materials with Effect of Memory of the Form (SMA) in the Conditions of a Plasma 
Dusting and their Optimisation for Maintenance of its Functional-mechanical Properties; Nova Science Publishers: New York, NY, USA, 2012; Volume 10, p. 375.

6. Blednova, Z.M.; Rusinov. P.O. Compositional Design of Surface Layers from Materials with the Effect of Shape Memory: Monograph. Publishing house of KubSTU, Krasnodar, Russia, 2017; p. 280.

7. Puzryakov, A.F. Theoretical Foundations of Plasma Spraying Technology. Publishing House of MSTU, N.E. Bauman: Moscow, Russia, 2003; p. 356.

8. Rusinov, P.O.; Blednova, Z.M. Promising materials and technologies: a collective scientific monograph. Features of the formation of multicomponent surface layers of materials with SME under high-energy influences; Chapter 8, pp. 158-178; A.V. Alifanov [et al.] // VSTU: Vitebsk, Belarus, 2015, 1, 398.

9. Johnson, N.; Lyon, F. Statistics and Experimental Design in Engineering and Science. Methods of Data Processing; Mir: Moscow, Russia, 1980; p. 511.

(C) 2020 by the authors. Submitted for possible open access publication under the terms and conditions of the Creative Commons Attribution (CC BY) license (http://creativecommons.org/licenses/by/4.0/). 